\title{
Symmetry-Driven Band Gap Engineering in Hydrogen Functionalized Graphene
}

Jakob Holm Jørgensen, ${ }^{\dagger}$ Antonija Grubišić Čabo, ${ }^{\dagger}$ Richard Balog, $^{\dagger \dagger}{ }^{\dagger}$ Line Kyhl, ${ }^{\dagger}$ Michael N. Groves, ${ }^{\dagger}$ Andrew Martin Cassidy, ${ }^{\dagger}$ Albert Bruix, ${ }^{\dagger}$ Marco Bianchi, ${ }^{\dagger}$ Maciej Dendzik, ${ }^{\dagger}$ Mohammad Alif Arman, ${ }^{\S}$ Lutz Lammich, ${ }^{\dagger}$ José Ignacio Pascual, $^{\ddagger}$ Jan Knudsen, ${ }^{\S, \| \odot ~ B j ø r k ~ H a m m e r, ~}{ }^{\dagger}$ Philip Hofmann, ${ }^{\dagger}$ and Liv Hornekaer* ${ }^{*} \dagger$

${ }^{\dagger}$ Department of Physics and Astronomy and Interdisciplinary Nanoscience Center iNANO, Aarhus University, Aarhus C DK-8000, Denmark

${ }^{\ddagger}$ CIC nanoGUNE and Ikerbasque, Basque Foundation of Science, Donostia-San Sebastian 20018, Spain

${ }^{\S}$ Division of Synchrotron Radiation Research and "The MAX IV Laboratory, Lund University, Box 118, Lund 22100, Sweden

Supporting Information

ABSTRACT: Band gap engineering in hydrogen functionalized graphene is demonstrated by changing the symmetry of the functionalization structures. Small differences in hydrogen adsorbate binding energies on graphene on $\operatorname{Ir}(111)$ allow tailoring of highly periodic functionalization structures favoring one distinct region of the moiré supercell. Scanning tunneling microscopy and X-ray photoelectron spectroscopy measurements show that a highly periodic hydrogen functionalized graphene sheet can thus be prepared by controlling the sample temperature $\left(T_{\mathrm{s}}\right)$ during hydrogen functionalization. At deposition temperatures of $T_{s}=645 \mathrm{~K}$ and above, hydrogen adsorbs
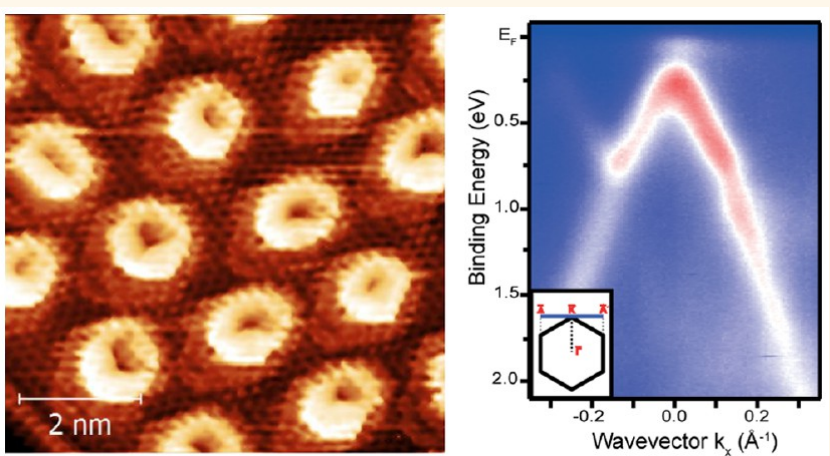
exclusively on the HCP regions of the graphene/ $\operatorname{Ir}(111)$ moire structure. This finding is rationalized in terms of a slight preference for hydrogen clusters in the HCP regions over the FCC regions, as found by density functional theory calculations. Angle-resolved photoemission spectroscopy measurements demonstrate that the preferential functionalization of just one region of the moire supercell results in a band gap opening with very limited associated band broadening. Thus, hydrogenation at elevated sample temperatures provides a pathway to efficient band gap engineering in graphene via the selective functionalization of specific regions of the moiré structure.

KEYWORDS: graphene, Ir(111), hydrogen, band gap engineering, functionalization, STM, photoemission spectroscopy

raphene is a promising candidate for applications in postsilicon electronics ${ }^{1}$ due to its remarkable electronic properties ${ }^{2}$ including a carrier mobility exceeding 200,000 $\mathrm{cm}^{2} \mathrm{~V}^{-1} \mathrm{~s}^{-1}$. However, graphene lacks an electronic band gap, and therefore graphene based electronic devices (e.g., field effect transistors based on graphene) cannot be switched completely off due to a high residual current. To overcome this disadvantage, band gap engineering in graphene has attracted tremendous research interest, and several different routes have been proposed in the literature. For example, cutting a graphene sheet into nanoribbons confines electrons in an additional dimension, which will in some cases open a band gap where the size scales inversely with the ribbon width. ${ }^{4}$ For the case of a $N=7$ graphene nanoribbon, a band gap of $2.3 \mathrm{eV}$ has been measured. ${ }^{5}$ A tunable gap in bi- and trilayer graphene with broken sublattice symmetry can be achieved by electrical gating. ${ }^{6-8}$ Band gaps with a size of up to $250 \mathrm{meV}$ have been demonstrated using this approach. Also, molecular adsorption onto a graphene sheet appears to be a promising route to achieve the desired gap opening. Physisorption of molecules onto graphene has been shown theoretically ${ }^{9}$ and experimentally ${ }^{10}$ to generate a gap via induced periodic modulation of the electronic structure, and the adsorption of water on the basal plane of graphene was found to induce a band gap of up to 206 meV. ${ }^{10}$ However, due to the weak van der Waals interaction between graphene and physisorbed adsorbates, such systems may become unstable at the temperatures used for semiconductor processing. ${ }^{11}$ A higher thermal stability of the adsorbates can be achieved through chemical functionalization

Received: July 13, 2016

Accepted: November 9, 2016

Published: November 9, 2016 


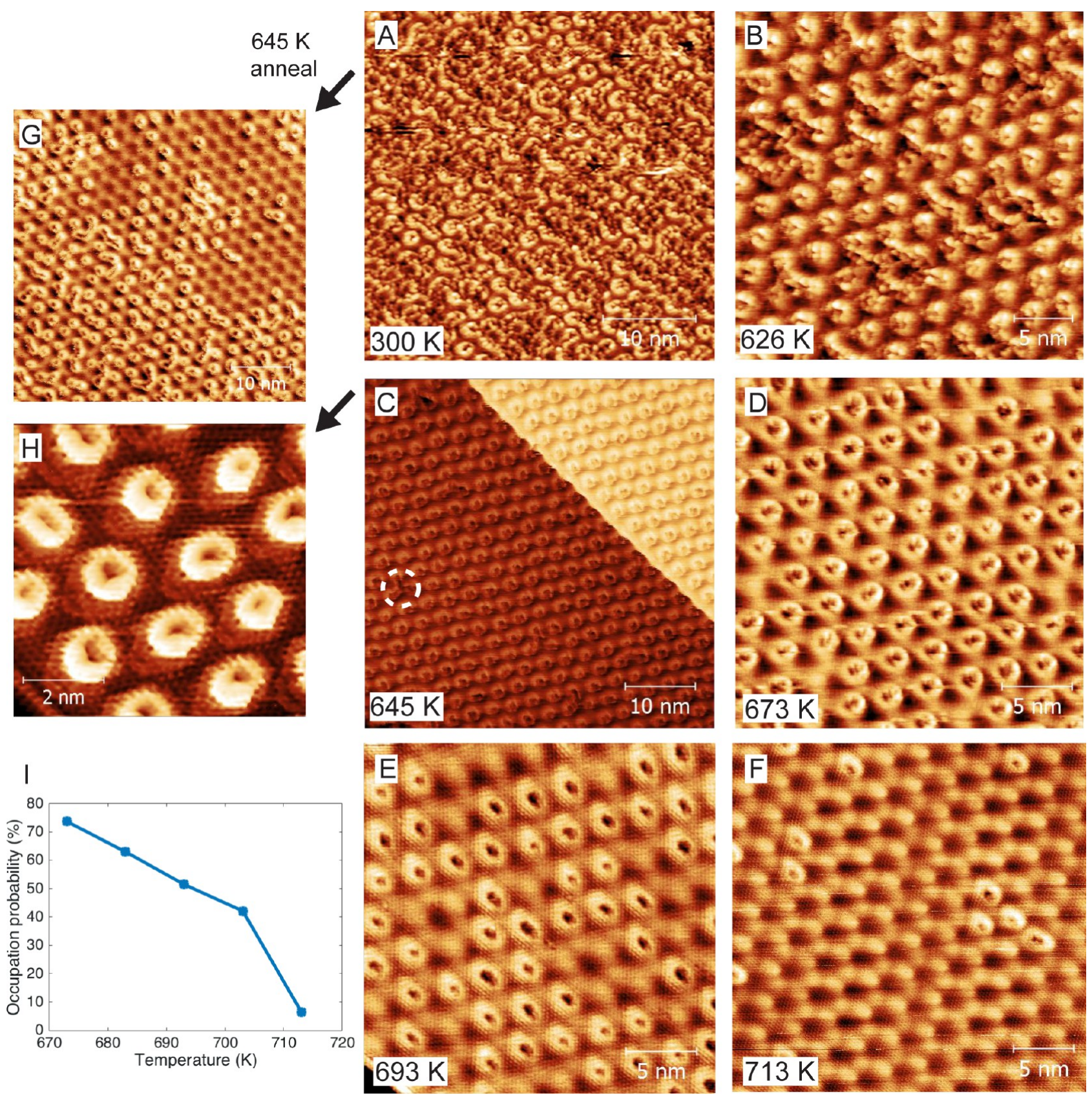

Figure 1. Hydrogenation of graphene on $\operatorname{Ir}(111)$. (A) STM image of graphene/Ir(111) saturated with atomic hydrogen at a sample temperature of $300 \mathrm{~K}$. Extended hydrogen structures following the moire periodicity are observed, $U=-1076 \mathrm{mV}, I=0.93 \mathrm{nA}$. (B) Graphene $/ \operatorname{Ir}(111)$ hydrogenated at a sample temperature of $626 \mathrm{~K}$. Isolated hydrogen clusters as well as extended structures are observed, $U$ $=-926 \mathrm{mV}, I=0.53 \mathrm{nA}$. (C) Hydrogenation at $645 \mathrm{~K}$. A highly periodic hydrogenation structure following the HCP regions of the moire structure is found with few defects, one of which is marked by a white dashed circle, $U=-285 \mathrm{mV}, I=0.69 \mathrm{nA}$. (D) Hydrogenation at $673 \mathrm{~K}$. Exclusively HCP regions of the moire structure are now hydrogenated. A few defects in the hydrogen pattern are observed, $U=-857 \mathrm{mV}, I=$ $0.54 \mathrm{nA}$. (E) Increasing the hydrogenation temperature to $693 \mathrm{~K}$ leads to an increased defect density in the hydrogen pattern, $U=-412 \mathrm{mV}, I$ $=0.78 \mathrm{nA}$. (F) At $713 \mathrm{~K}$, only a few hydrogen clusters are observed, $U=-427 \mathrm{mV}, I=0.65 \mathrm{nA}$. (G) STM image of graphene/Ir(111) hydrogenated at RT followed by an anneal to $645 \mathrm{~K}$ for $1 \mathrm{~min}$. The resulting hydrogen structure is found to be disordered, consisting of areas of high coverage as well as hydrogen-free areas, $U=309 \mathrm{mV}, I=0.30 \mathrm{nA}$. (H) Atomically resolved image of graphene hydrogenated at a sample temperauture of $645 \mathrm{~K}$. Areas of pristine graphene are found between the hydrogen clusters, $U=256 \mathrm{mV}, I=1.2 \mathrm{nA}$. (I) The probability of populating an HCP region with a hydrogen cluster was estimated from the STM images as a function of deposition temperature. The population probability decreases linearly with deposition temperature up to $\sim 700 \mathrm{~K}$. Above this temperature, a rapid decrease is observed.

with atoms or molecules. ${ }^{12}$ In this regard, functionalization of graphene by atomic hydrogen has been extensively studied both experimentally $^{13-16}$ and theoretically. ${ }^{17-20}$ A fully hydrogenated sheet of graphene, with hydrogen bound to every carbon atom on alternative sides of the graphene plane, has been coined graphane. ${ }^{21}$ Calculations predict that such a material will be a wide gap insulator with a gap size as high as $5.4 \mathrm{eV} .{ }^{22}$ Balog et al. ${ }^{13}$ showed that a reasonably large band gap 

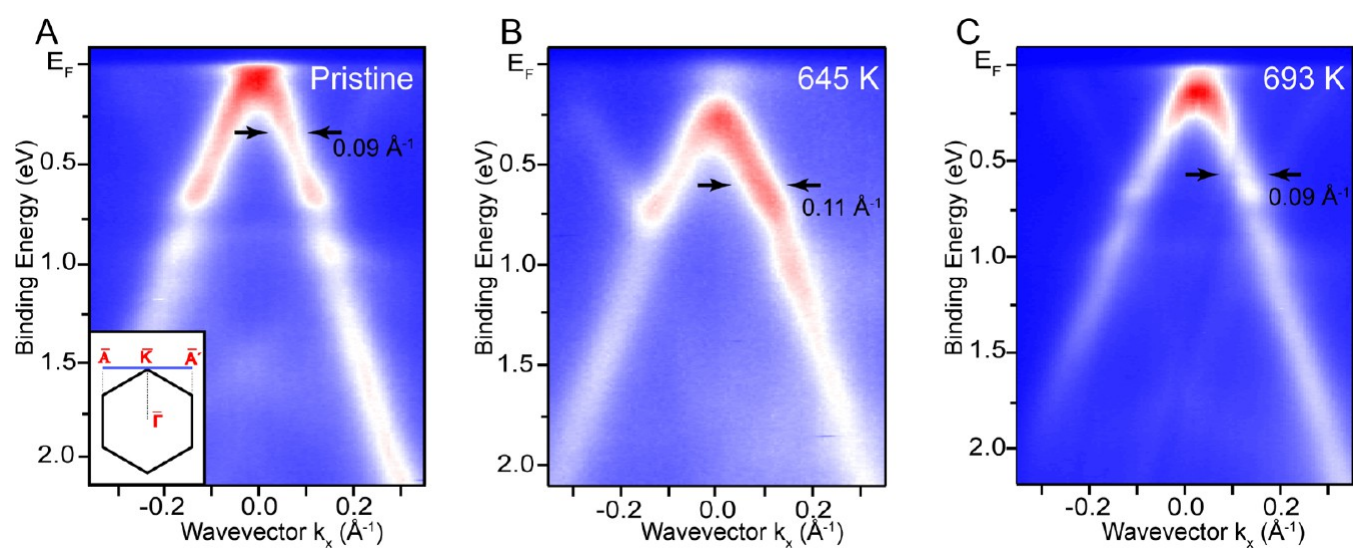

Figure 2. Angle-resolved photoemission spectroscopy measurements of (A) clean graphene/Ir(111). Graphene hydrogenated at a sample temperature of (B) $645 \mathrm{~K}$ and (C) at $693 \mathrm{~K}$, showing a band gap of at least 290 and $146 \mathrm{meV}$, respectively. The spectra are cuts through the $\mathrm{K}$ point along the $\bar{A}-\bar{K}-\bar{A}^{\prime}$ direction of the Brillouin zone, as indicated in the inset. Positions where the widths of the momentum distribution curves were measured are indicated by the arrows in the figure along with the extracted values.

with a size of at least $450 \mathrm{meV}$ can be opened in graphene through the formation of a periodic pattern of chemisorbed hydrogen atoms. This was achieved by exposing a graphene sheet, grown by chemical vapor deposition (CVD) on an $\operatorname{Ir}(111)$ surface, to a flux of hydrogen atoms at room temperature (RT). However, under these conditions, the formation of imperfect hydrogen structures with increasing hydrogen coverage leads to a pronounced scattering of the charge carriers, and therefore a significant broadening of the $\pi$ band was also observed. Likewise, oxygenated graphene/ $\operatorname{Ir}(111)^{23}$ and hydrogenated quasi-free-standing graphene on $\mathrm{Ni}(111)^{14}$ show a substantial gap in the $\pi$-band, but as in the previous case, the functionalized patterns are imperfect, and the gap opening is accompanied by undesired band broadening.

In this study, we demonstrate a symmetry-driven band gap engineering in hydrogenated graphene/Ir(111). Small differences in thermal stability of hydrogen adsorbate structures on graphene/ $\operatorname{Ir}(111)$ are used to drive a transition from a hydrogen functionalization superlattice with honeycomb symmetry to one with a rotated triangular symmetry. ${ }^{24}$ The rotated triangular hydrogen functionalization superlattice is highly periodic leading to significantly reduced electron scattering and thus limited band broadening. Two different approaches for tuning the hydrogen adsorption structures and the resulting band gap are explored in this paper: (1) hydrogenation while keeping the sample at elevated temperatures and (2) hydrogenation at RT followed by annealing the sample. Using scanning tunneling microscopy (STM), we find that a highly periodic array of graphane-like hydrogen clusters can be obtained with the first approach. The size of the most stable clusters formed at elevated temperatures has been estimated by comparing STM and density functional theory (DFT) calculations. The STM measurements reveal that the chemisorbed hydrogen atoms in graphene-like clusters occupy only a single site of the moiré supercell, namely the HCP site. Angle-resolved photoemission spectroscopy (ARPES) was carried out for the samples hydrogenated at elevated temperatures, revealing a correlation between size of the band gap and the deposition temperature, thus demonstrating tunability of the gap in the graphene/Ir(111) system. Furthermore, the high periodicity in these structures was seen to result in only very limited band broadening.

\section{RESULTS AND DISCUSSION}

Single crystal graphene was grown on $\operatorname{Ir}(111)$ by combining temperature-programmed growth (TPG) and CVD methods. ${ }^{25}$ This yields a continuous layer of graphene spanning the Ir terraces and step edges consistent with a single crystal sheet as reported elsewhere, ${ }^{26}$ see Figure S1 in Supporting Information.

The clean graphene sample was subsequently exposed to atomic hydrogen at various surface temperatures $\left(T_{\mathrm{s}}\right)$ ranging from RT up to $713 \mathrm{~K}$. Hydrogen saturation coverage was obtained by $\mathrm{H}$ deposition at $\mathrm{RT}$ with the resulting structure shown in the STM image in Figure 1A. The hydrogen adsorbs in a quasi-periodic structure following the moire periodicity and comprises graphane-like- and dimer structures. ${ }^{13,16}$ Hydrogenation at $T_{s}=626 \mathrm{~K}$ yields a structure exhibiting increased ordering, as seen in Figure 1B. Isolated hydrogen clusters following the periodicity of the moire structure are now found abundantly on the surface. These are attributed to graphanelike structures in the HCP region of the moiré supercell (in this region, the center of the graphene hexagons is located above an HCP site in the $\operatorname{Ir}(111)$ surface, as illustrated in Figure 5C), details on the assignment are given below. Additionally, extended hydrogen structures are present on the surface due to simultaneous population of neighboring FCC and HCP regions. Increasing the deposition temperature to $T_{s}=645 \mathrm{~K}$ results in a highly periodic hydrogen structure, as seen in Figure 1C. Mixed site occupancy within the moiré (i.e., hydrogen in both HCP and FCC regions) is no longer present, and only single site occupancy of HCP regions is observed, giving rise to a very well-defined hydrogen pattern exhibiting a triangular symmetry. The hydrogen clusters now appear as isolated donut-like structures pinned to specific parts of the moiré supercell. Some defects in the hydrogen pattern, one indicated by a dashed circle in Figure 1C, are found for this deposition temperature, but the occurrence of these is relatively rare even in large area scans and at various positions on the sample. Therefore, such a hydrogen pattern can be considered highly periodic. An atomically resolved image of this structure is shown in Figure $1 \mathrm{H}$. In this image, pristine graphene is observed in-between the hydrogen clusters, which demonstrates that hydrogen binds exclusively on a specific region on the moire superlattice. Increasing the sample temperature to $T_{\mathrm{s}}$ $=673 \mathrm{~K}$ during hydrogen deposition leads to the appearance of vacancies, i.e., missing clusters, in the hydrogen pattern, Figure 

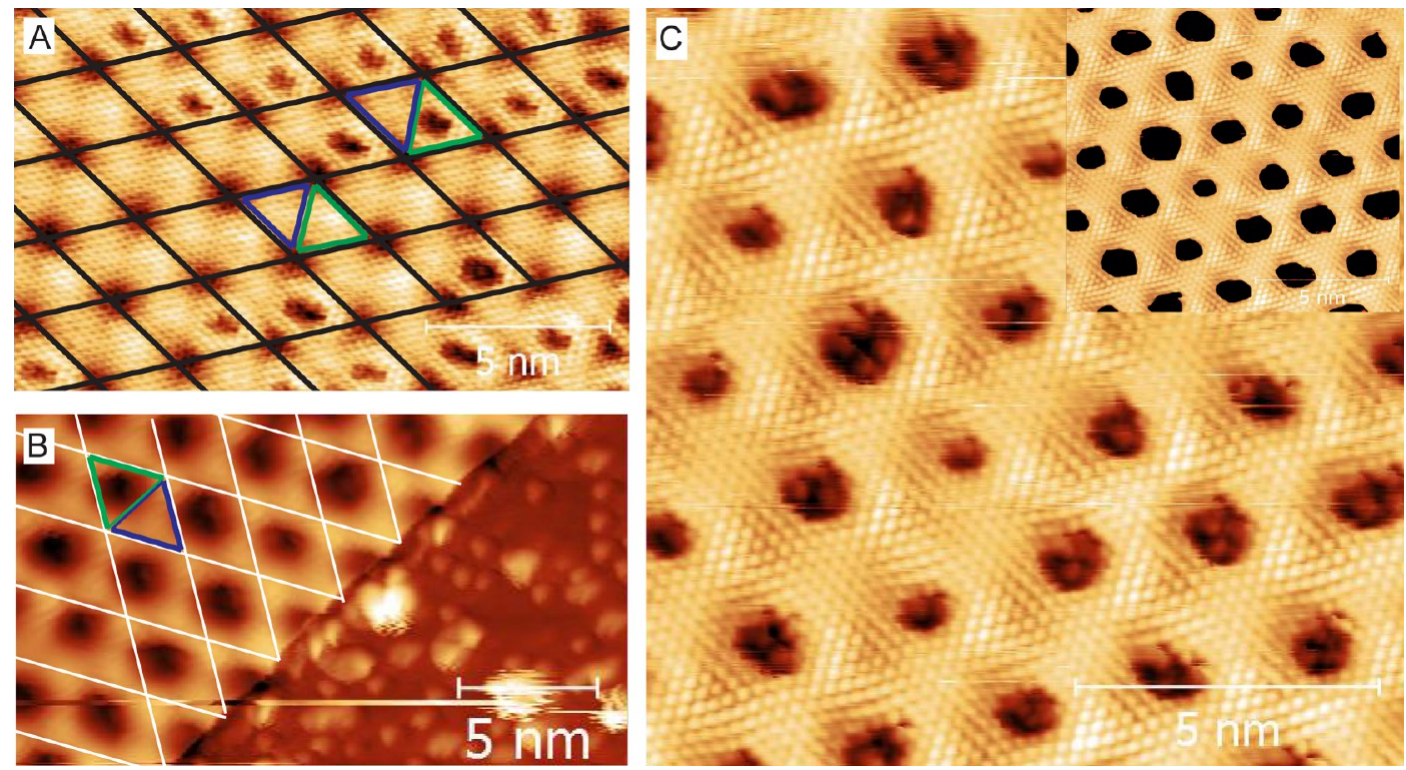

Figure 3. (A) STM image of partially hydrogenated graphene/ $\operatorname{Ir}(111)$ formed at $693 \mathrm{~K}$. In the hydrogen-free areas, it is possible to identify moiré regions based on the STM apparent height. From the overlaid lattice, we are able to assign the hydrogenation regions to the HCP region (green triangle) on the moire superlattice. No hydrogen clusters are observed in the FCC regions (blue triangle), $U=-159 \mathrm{mV}, I=$ $0.73 \mathrm{nA}$. (B) STM image of submonolayer graphene. The graphene domain is found in the left part of the image and (polluted) iridium in the right part. From the jagged zigzag interface, the moiré regions can be determined. The hydrogen clusters (appearing dark in the image) are found exclusively in the half unit cell pointing away from the interface, confirming the adsorption site to be $\mathrm{HCP}, U=338 \mathrm{mV}, I=0.23 \mathrm{nA}$. (C) STM image of graphene hydrogenated at $645 \mathrm{~K}$. Hydrogen clusters appear as depressions. The cluster area, corresponding to the black areas in the inset, was measured on this image yielding a cluster coverage of $18 \%$.

1D. Elevating the deposition temperature further sees more defects appear in the hydrogen cluster pattern, leaving increased areas of clean graphene as seen in Figure 1E,F for sample deposition temperatures of 693 and $713 \mathrm{~K}$, respectively. The fraction of populated HCP regions has been estimated based on STM images for hydrogenation temperatures of $T_{\mathrm{s}}=$ $673 \mathrm{~K}$ and above. The observed hydrogen cluster occupation probability for HCP regions is plotted as a function of hydrogenation temperature in Figure 1I, showing a linear decrease up to a temperature of approximately $700 \mathrm{~K}$ above which the probability rapidly decays to zero in agreement with previous X-ray photoelectron spectroscopy (XPS) results. ${ }^{27}$

For comparison, we explored the possibility of forming the highly periodic hydrogen pattern by postannealing of the hydrogen-saturated graphene sheet. After deposition of hydrogen at $T_{\mathrm{s}}=\mathrm{RT}$ (Figure $1 \mathrm{~A}$ ), the sample was annealed to $645 \mathrm{~K}$ for $1 \mathrm{~min}$ in order to desorb the least stable structures. ${ }^{27}$ The resulting hydrogen pattern is shown in Figure 1G. Areas exhibiting a periodic hydrogen pattern are now present, consistent with the high temperature deposition results. However, large hydrogen structures covering both HCP and FCC regions, together with relatively large patches of clean graphene are found, resulting in an overall disordered surface. Furthermore, we find that annealing the hydrogen-saturated sample to various temperatures in the vicinity of $645 \mathrm{~K}$ did not improve the hydrogen patterning, and it is therefore concluded that an extended periodic structure cannot be prepared using this procedure.

ARPES measurements were performed at the SGM3 beamline of ASTRID $2^{28}$ to determine how the hydrogenation changes the electronic structure of graphene. Figure 2 displays ARPES spectra for hydrogen depositions at various temperatures. The spectrum of clean graphene/ $\operatorname{Ir}(111)$ taken along the $\bar{A}-\bar{K}-\bar{A}^{\prime}$ direction is shown in Figure 2A. Mini gaps and replica bands are visible as a consequence of the moiré superstructure. $^{29,30}$ The width of a momentum distribution curve (MDC) cut through the $\pi$-band measured at an energy of $400 \mathrm{meV}$ below the interpolated Dirac point is found to be 0.09 $\AA^{-1}$. ARPES spectra of graphene hydrogenated at sample temperatures of 645 and $693 \mathrm{~K}$ are shown in Figure 2B,C, respectively. In both cases, the mini gaps and replica bands are preserved. The observation of well-defined cones and mini gaps in hydrogenated samples confirms a well-ordered hydrogen pattern. Importantly, the evolution of a band gap at different hydrogenation temperatures is clearly visible: Energy distribution curve (EDC) analysis of the spectra (see Methods section) reveals that for hydrogenation at $T_{\mathrm{s}}=645 \mathrm{~K}$, the top of the valence band is at $290 \mathrm{meV}$ below the Fermi level. Since ARPES measurements probe only occupied states, this demonstrates the opening of a band gap of at least $290 \mathrm{meV}$. For hydrogenation at $T_{\mathrm{s}}=693 \mathrm{~K}$, displayed in Figure 2C, a band gap opening of at least $146 \mathrm{meV}$ is observed. MDC band widths measured at an energy of $400 \mathrm{meV}$ below the interpolated Dirac point energy show only very limited additional broadening for graphene hydrogenated at these temperatures. In contrast, a band gap of at least $450 \mathrm{meV}$ can be achieved for RT hydrogenation. ${ }^{13}$ This, however, comes at the expense of a greatly increased MDC width. Thus, the results presented here demonstrate that the size of the hydrogen induced band gap in graphene can be engineered by varying the sample temperature during the hydrogen deposition. Furthermore, the MDC width of the $\pi$-band is found to increase only slightly compared to clean graphene for the highly periodic hydrogenated samples formed at high deposition temperature, indicating a negligible influence on the scattering of charge carriers.

To identify the preferential adsorption site, an area of partial hydrogen coverage was investigated as depicted in the STM 
image of Figure 3A. It has previously been established that the different high-symmetry sites of the moire structure can be identified based on the STM apparent height in most common imaging modes. ${ }^{26,31}$ The dark depressions correspond to ATOP regions, while the bright areas are $\mathrm{HCP} / \mathrm{FCC}$ regions, with the HCP region being slightly brighter (see Figure 5C for an illustration of the positions of the regions on the moire supercell). These regions have been visualized in the STM image in Figure $3 \mathrm{~A}$ with the green and blue triangles representing HCP and FCC regions, respectively. The hydrogen clusters, imaged as dark depressions, are only found in the green triangles. On the basis of this, we find that hydrogen clusters exclusively populate HCP regions at deposition temperatures of $645 \mathrm{~K}$ and above. To further support this assignment, and to ensure that our image mode is similar to the ones in refs 26 and 31, a submonolayer of graphene was hydrogenated at $T_{s}=645 \mathrm{~K}$. In the STM image of Figure 3B, a graphene island is found along an Ir step, forming a jagged zigzag edge. ${ }^{31}$ It has previously been established that the graphene areas protruding toward the Ir are ATOP regions and that HCP (FCC) regions are identified as the triangular half unit cells pointing away from (toward) the interface. ${ }^{31}$ In Figure 3B, a grid has been drawn identifying the moire unit cell with the ATOP regions at line-intersections. The hydrogen clusters are found only in the half unit cells pointing away from the boundary (example indicated by the green triangle), while the half unit cells pointing toward the interface (exemplified by the blue triangle) do not contain hydrogen. This confirms that the hydrogen clusters are adsorbed only on the HCP regions of the moire structure.

Since the individual hydrogen atoms are not resolved in the STM images, it is not straightforward to determine the number of atoms in each cluster, however, an estimate can be made by considering the cluster area. This was done using the STM image in Figure 3C, which displays graphene hydrogenated at $645 \mathrm{~K}$, with the hydrogen clusters imaged as depressions. The inset of Figure $2 \mathrm{C}$ shows the hydrogen affected area to be roughly $18 \%$. It is estimated that in this area, approximately half of the carbon atoms (every second atom) bind to hydrogen resulting in a $\sim 9 \%$ coverage, while the remaining carbon atoms within the cluster are bound to surface Ir atoms. ${ }^{12,13,16}$ The alternating bond formation to hydrogen and iridium means that the carbon atoms in hydrogen clusters are $\mathrm{sp}^{3}$ hybridized. Therefore, the clusters exhibit a graphane-like nature and are expected to be highly insulating and are thus expected to behave like holes in the graphene sheet when considering how the electronic structure is modified.

A periodic array of holes in a graphene sheet is known as a graphene antidot lattice, and the electronic effects of honeycomb, triangular, and square antidot lattices have been studied theoretically. ${ }^{24,32}$ Theory predicts that graphene patterned with a triangular antidot lattice acquires a global band gap which scales with the lattice and hole size according to the universal scaling equation: ${ }^{32}$

$$
E_{\mathrm{g}} \approx K \times \frac{\sqrt{N_{\text {removed }}}}{N_{\text {total }}}
$$

where $K$ is a proportionality constant in $\mathrm{eV}, N_{\text {total }}$ is the number of carbon atoms in the antidot unit cell, and $N_{\text {removed }}$ is the number of atoms removed to form the hole. For honeycomb and rotated triangular lattices, however, the gap behavior is nontrivial and only appears for certain lattice parameters. ${ }^{24}$ This closing and opening of a gap has been explained by a Clar sextet representation which reveals gap formation whenever only one fully benzenoid structure can be formed and vanishing or a very small gap otherwise. ${ }^{24}$ However, this nontrivial behavior appears to be valid only for perfect antidot lattices. For example, it has been shown that hydrogen clusters positioned in a honeycomb superstructure still follow the universal scaling equation when the size and shape of hydrogen clusters vary randomly from cell to cell. ${ }^{33}$ This is in accordance with earlier experimental results where a large gap opening for this structure was found. ${ }^{13}$ In contrast to previous experiments, the hydrogenation at high $T$ demonstrated here generates a rotated triangular superlattice. The tight binding model predicts zero gap for such a superlattice when the lattice parameters are given by the graphene-Ir(111) moire. $^{24}$ Despite this, our ARPES data clearly show significant gap formation. We therefore propose that, similar to the honeycomb superstructure, a rotated triangular superlattice with imperfect hydrogen clusters (as seen in Figure 3c) may recover the scaling law behavior.

By using the average number of $\mathrm{C}$ atoms in a cluster determined from Figure $3 \mathrm{C}$, we find the ratio $\sqrt{N_{\text {removed }}} / N_{\text {total }}$ to be 0.029 . For the rotated triangular lattice, a $K$ value of approximately $23 \mathrm{eV}$ can be extracted from ref 24, yielding a predicted band gap of $667 \mathrm{meV}$. In the ARPES data, the measured distance between the band maximum and the Fermi energy of $290 \mathrm{meV}$ represents a lower bound for the gap. Assuming zero doping, the full gap may approach twice the measured ARPES value, which would be in reasonable agreement with the prediction. Additionally, the $K$ value may be overestimated since it is based on parameters for perfect graphene. $^{33}$

The hydrogen functionalization at different sample temperatures has been followed with high resolution X-ray photoelectron spectroscopy (HR-XPS). The C 1s spectrum of graphene acquired before and after hydrogen exposure to saturation at RT is shown in Figure 4A,B, respectively. After hydrogenation, the spectrum can be deconvoluted with four components assigned to $\mathrm{C}$ atoms: located in clean but doped graphene $\left(C_{c}\right)$, located within the graphane-like clusters $\left(C_{b}\right.$ and $\mathrm{C}_{\mathrm{d}}$ ), and finally located in dimers ${ }^{34}$ and more disordered clusters $\left(C_{a}\right)$, in accordance with previously published studies. ${ }^{27}$ The $C_{a}$ component is observed to be significant in this case. Details about the deconvolution are given in the Supporting Information. In Figure 4C,D, C 1s spectra obtained for samples hydrogenated at 626 and $645 \mathrm{~K}$ are displayed. Here the $\mathrm{C}_{\mathrm{b}}$ and $\mathrm{C}_{\mathrm{d}}$ components associated with graphane-like structures and clean graphene clearly dominate, while the $\mathrm{C}_{\mathrm{a}}$ component has disappeared. This is in perfect agreement with the STM measurements showing a decrease in the complexity of the hydrogen adsorbate structures, with preferential population of HCP regions at these temperatures. For the $645 \mathrm{~K}$ deposition, a decrease in the $\mathrm{C}_{\mathrm{b}}$ and $\mathrm{C}_{\mathrm{d}}$ components and an increase in the $\mathrm{C}_{c}$ component are observed, consistent with the STM measurements showing a reduction of hydrogen coverage at this temperature.

The origin of the preference for hydrogenation of the HCP regions was investigated by DFT. The adsorption energy gained per hydrogen atom when a graphane-like hydrogen cluster forms on the basal plane of graphene/ $\operatorname{Ir}(111)$, as determined by DFT calculations, is displayed in Figure 5A. The adsorption energy was calculated for single-site occupancy of either HCP 


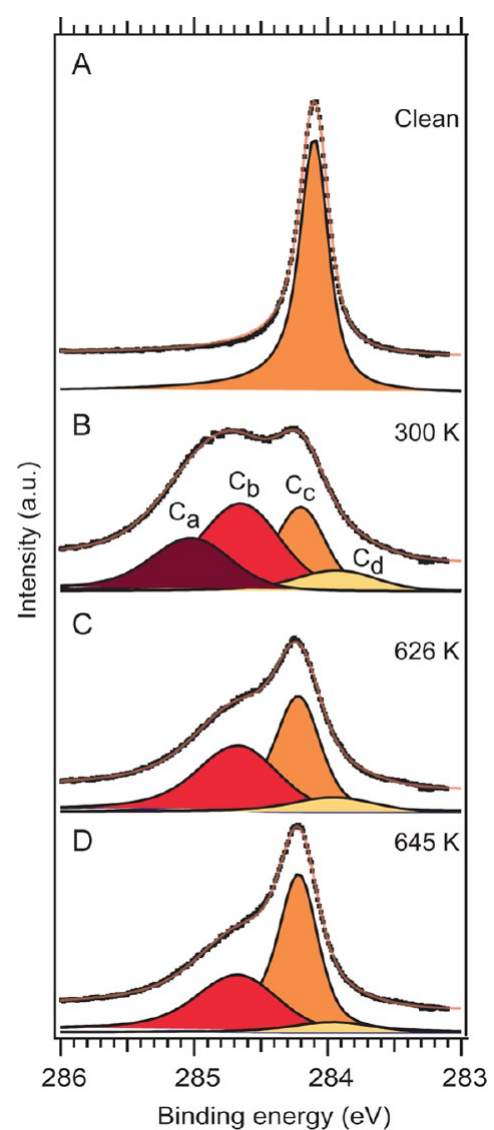

Figure 4. C 1s XPS spectra of graphene/Ir(111). (A) pristine graphene/Ir(111) sample. Graphene/Ir(111) exposed to hot atomic hydrogen with the sample at (B) $300 \mathrm{~K}$, (C) $626 \mathrm{~K}$, and (D) $645 \mathrm{~K}$. All spectra were acquired at RT. Peak components are identified as follows: $C_{c}$ is unperturbed $C$ atoms in the graphene lattice; $C_{b}$ and $C_{d}$ are related to the formation of graphane-like clusters; and $\mathrm{C}_{a}$ is ascribed to more disordered adsorbate structures, including hydrogen dimers on the graphene sheet. Clearly the $\mathrm{C}_{\mathrm{a}}$ component is absent for hydrogenation at increased sample temperatures, consistent with the presented STM data.

or FCC regions (blue and red curves, respectively) as well as for mixed occupancy with equal size clusters on both the FCC and HCP regions (green curve). A smaller $8 \times 8$ graphene unit cell (compared with the full $10.32 \times 10.32$ cell) was used to facilitate the calculations. Justification for the validity of this smaller unit cell can be found in Supporting Information. The calculations show that hydrogen adsorption onto sites in the HCP and FCC regions is almost equally stable, with sites in the HCP region being slightly more favorable. The most stable single-site adsorption structures are found for a coverage of approximately $6-10 \%$, corresponding to $7-12$ atoms in the smaller unit cell used. For this low hydrogen coverage, mixed occupancy is found to be significantly less stable compared to single-site occupancy. This can be ascribed to the fact that addition of the first hydrogen atom forces the graphene sheet to move closer to the Ir surface. ${ }^{13}$ But once this has been accomplished, the addition of subsequent hydrogen atoms to the cluster results in increased average binding energies and hence more stable structures. The graphane-like cluster formation is facilitated by the specific registry at the HCP and FCC regions between the graphene and iridium surface atoms, where every second carbon atom is placed right above an iridium atom. The addition of $\mathrm{H}$ atoms continues until this

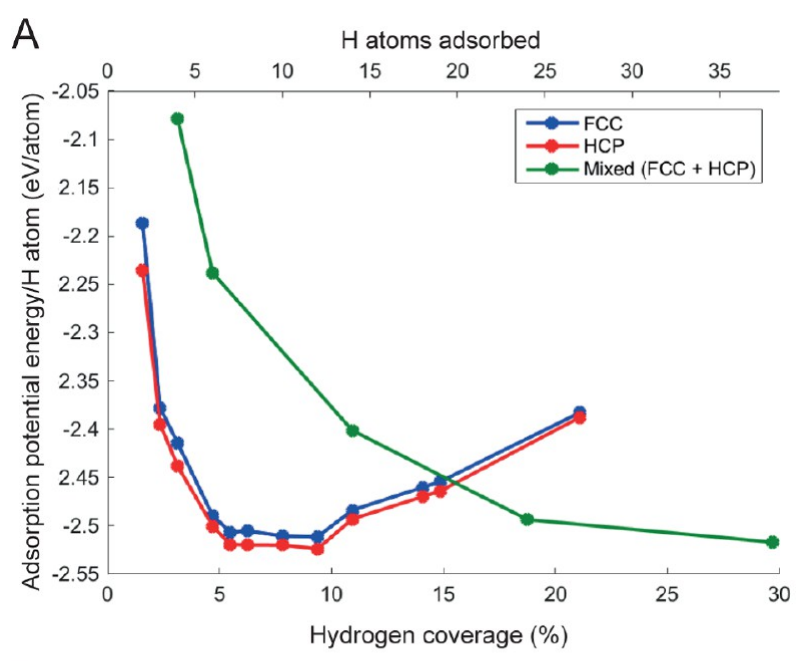

B C

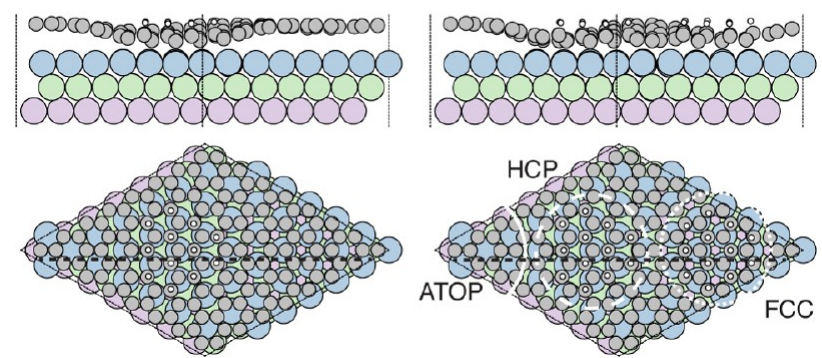

Figure 5. (A) DFT calculated average binding energies per adsorbed $\mathrm{H}$ atom as a function of the hydrogen coverage/number of $\mathrm{H}$ atoms in each cluster using an $8 \times 8$ graphene unit cell on a 7 $\times 7 \operatorname{Ir}(111)$ surface. Hydrogen clusters adsorbed in the FCC and HCP regions of the moire structure correspond to the red and the blue curves, respectively, and show a very similar behavior with only a small offset. The green curve corresponds to adsorption of equal amounts of $\mathrm{H}$ atoms on both the FCC and the HCP regions. It is seen that single site occupancy is the most favorable configuration for low hydrogen coverage, whereas mixed occupancy becomes increasingly favorable at higher coverage. (B) DFT derived structure for a 12 atom hydrogen cluster on the HCP region of graphene $/ \operatorname{Ir}(111)$. (C) DFT derived structure for two 12atom hydrogen clusters on the FCC region and the HCP region of graphene/Ir(111). The ATOP, HCP, and FCC regions have been marked by a full, dashed , and dotted circle, respectively. In (B) and (C), the side views were cut along the black dashed lines marked in the top view models.

registry breaks down as seen in Figure 5B. As a result, an increased binding energy is found for mixed occupancy of both sites at higher hydrogen coverage reaching an optimal value at $30 \%$ coverage.

This situation is in accordance with the presented STM images, where mixed site occupancy is observed for hydrogen saturation coverages obtained at RT (Figure 1A). As the hydrogen saturated graphene is annealed to $645 \mathrm{~K}$, the less stable adsorption configurations disappear resulting in ordered hydrogen clusters on the HCP regions as well as large clean areas (Figure 1G). In the case of hydrogen exposure at increased substrate temperature, the system is forced to form only the most stable hydrogen-configurations resulting in a well-ordered hydrogen pattern following the HCP regions of the moire superlattice (Figure 1C). Furthermore, it should be noted that each of the two clusters in a "mixed" configuration is 

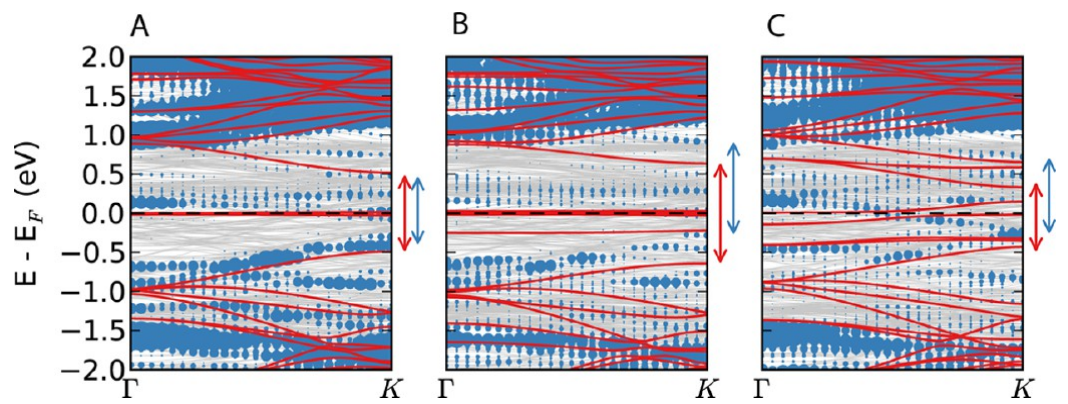

Figure 6. Band dispersion for hydrogenated graphene on $\operatorname{Ir}(111)$ for $(A)$ three hydrogen atoms adsorbed in the HCP region of the moiré, (B) six hydrogen atoms adsorbed on the HCP region, and (C) three hydrogen atoms adsorbed in each of the HCP and FCC regions. The bands for each structure are shown in light gray on a white background. The contribution from carbon $2 \mathrm{p}_{z}$ orbitals to each eigenvalue is proportional to the size of the blue dots, which therefore indicate the graphene-like character of each band. The red lines correspond to the band structure of the same hydrogen/graphene structure but without the Ir slab. Arrows have been added to indicate the corresponding band gaps.

less stable than a similar sized cluster would have been for single site occupancy (e.g., compare the binding energy for a $3 \mathrm{H}$ atom cluster with single site occupancy with that of two $3 \mathrm{H}$ atom clusters, i.e., $6 \mathrm{H}$ atom coverage, for mixed site occupancy). This difference is ascribed to the finding that placing a hydrogen cluster on each of the HCP and FCC regions leads to a large buckling of the hydrogen-free graphene area in-between and therefore induces strain (Figure 5C). This strain can be relieved by adding additional hydrogen atoms to the area, leading to the merging of the individual clusters, in agreement with STM data. In the latter, in fact, a site of mixed occupation is typically imaged as a continuous cluster rather than two isolated ones, as can be seen in Figure 1A,B. Thus, despite the fact that the graphene and iridium atoms are not in registry in the in-between region, according to the STM images, hydrogen adsorption appears to be energetically favorable as a means of strain relief. The size of the most stable clusters for single site occupancy is captured nicely by the calculations. The energy minimum is found at a hydrogen coverage of $6-10 \%$, while the coverage found from the STM experiments is roughly $9 \%$. Also the relatively large spread in cluster sizes, seen in the STM image in Figure 3C, is in agreement with the broad minimum obtained by the calculations.

The calculations only show a slightly increased stability for hydrogen clusters on the HCP regions as compared to the FCC regions, roughly $20 \mathrm{meV}$ per hydrogen atom for the most favorable structures. However, a larger difference is observed for the addition of the first two $\mathrm{H}$ atoms in each cluster, where a binding energy difference of approximately $50 \mathrm{meV}$ per $\mathrm{H}$ atom is found. As this energy difference may be sufficient to ensure preferred population of the HCP region over the FCC region even for RT hydrogen atom deposition, the STM observation of population of both sites under these conditions is suggestive of the structures formed being governed by kinetic effects. Furthermore, the STM measurements displayed in Figure $1 \mathrm{G}$ show that annealing these structures to a higher temperature only results in a limited degree of increased order, presumably because diffusion of hydrogen atoms on the graphene/ $\operatorname{Ir}(111)$ surface is limited. On the other hand, during deposition at elevated temperatures, repeated adsorption/ desorption events allow for the formation of only the most stable structures on the surface, in accord with the STM observations displayed in Figure 1C. This behavior is somewhat different from that observed for metal clusters on graphene/ $\operatorname{Ir}(111)$ : While metal clusters also form preferentially in the HCP regions of the graphene/Ir(111) moire ${ }^{31}$ following a similar rehybridization mechanism as hydrogenated graphene/ $\operatorname{Ir}(111)$, this has been observed to occur preferentially at low temperatures. $^{31,35}$ This difference may well be ascribed to variations in diffusion barriers between the two systems as well as the strong interatomic metal bonds in the metal clusters favoring large clusters at high temperature.

The changes to the graphene/Ir band structure induced by hydrogenation were calculated using DFT. The band structures were obtained for three different hydrogenation cases: (a) three $\mathrm{H}$ atoms adsorbed in the $\mathrm{HCP}$ region of the moire, (b) six $\mathrm{H}$ atoms adsorbed in the HCP region, and (c) three $\mathrm{H}$ atoms adsorbed in each of the HCP and FCC regions resulting in a mixed occupancy. The structures can be seen in Supporting Information Figure S3. The corresponding band dispersions are displayed in Figure $6 \mathrm{~A}-\mathrm{C}$, respectively. The bands for each structure are shown in light gray on a white background. The contribution of $\mathrm{C} 2 \mathrm{p}_{z}$ orbitals to each eigenvalue is proportional to the size of the plotted blue dots, indicating the graphene-like character of each band. It should be noted that hybridization with Ir partially delocalizes graphene states, which impedes unambiguously determining the position of the $\pi$-bands. To more easily identify the $\pi$-bands in graphene on Ir, we also include the band structure of the hydrogenated freestanding graphene (HFSG) system resulting from removing the Ir slab from the optimized structures (red lines). The HFSG structures are not reoptimized after removal of Ir and therefore retain the same hydrogen configuration and graphene corrugation.

For single site occupancy on graphene/Ir, the $\pi$-bands are slightly upshifted with respect to HFSG. The shift is most likely induced by doping effects. The position of the filled $\pi$-band at $K$ with respect to the Fermi level is found to be $-0.30 \mathrm{eV}$ and $-0.28 \mathrm{eV}$ for 3-atom and 6-atom $\mathrm{H}$ clusters respectively. This is in reasonable agreement with the presented ARPES data in Figure $2 \mathrm{~B}$. We note, however, that for the calculated bands, the overall hydrogen coverage represents only $5.5 \%$ for the $6 \mathrm{H}$ case and is thus smaller than the one estimated for the ARPES data. The estimated size of the gap in hydrogenated graphene/Ir is slightly reduced compared to HFSG but remains significant. This result fully supports the experimental observations. Since many different effects such as cluster shape, ${ }^{33}$ symmetry of the hydrogen structure superlattice, ${ }^{24}$ and symmetry breaking between carbon atoms ${ }^{36}$ influence the formation of the gap, separating their individual contributions to the gap opening for the experimentally observed structures is beyond the scope of this article. We can however conclude that, while calculations predict that perfect rotated triangular as well as honeycomb 
antidot lattices may not open a gap in graphene, any deviation from the perfect structures induces a gap. For the same hydrogen superlattice, the size of the band gap is found to increase with $\mathrm{H}$ coverage in agreement with previous reports. ${ }^{13,24}$ However, the size of the gap seems to depend on the hydrogen superlattice symmetry. For the case of singlesite occupancy of the moire superlattice (rotated triangular lattice), the estimated gaps are 1.05 and $1.20 \mathrm{eV}$ for three $\mathrm{H}$ and six $\mathrm{H}$ clusters, whereas for the mixed occupancy, (honeycomb lattice) a band gap of $0.74 \mathrm{eV}$ is found. Additionally, mid gap states are observed in the HFSG due to the presence of unpaired electrons. We note that considering spin-polarization for the HFSG would lead not only to equivalent $\pi$-band gaps but also to nondegeneracy and slight energy shifts of the mid gap states. The latter seem to persist even in the presence of the Ir slab, although interaction with the metal surface strongly delocalizes them. The mid gap states for the mixed occupancy have stronger dispersion and are also more affected by the interaction with Ir, seemingly leading to the formation of new bands around the Fermi level.

\section{CONCLUSION}

In conclusion, we have demonstrated a hydrogen functionalization symmetry-driven approach to band gap engineering in graphene. A transition from a hydrogenation structure with honeycomb symmetry to one with rotated triangular symmetry is observed for increased functionalization temperatures. Hightemperature hydrogenation of graphene on $\operatorname{Ir}(111)$ at a sample temperature of $645 \mathrm{~K}$ leads to the formation of a highly periodic rotated triangular hydrogen pattern with the moire superstructure as a template. In this case, hydrogenation is found to occur exclusively on a specific single region in the moire unit cell, namely the HCP region, resulting in localized hydrogen clusters with $25 \AA$ periodicity. When lowering the hydrogenation temperature slightly to $T_{s}=626 \mathrm{~K}$, both FCC and HCP regions are hydrogenated, leading to a hydrogenation structure with honeycomb symmetry. On the other hand, an increase in hydrogenation temperature above $645 \mathrm{~K}$ leads to hydrogen cluster vacancies in the otherwise perfectly periodic rotated triangular hydrogen pattern. These observations are supported by DFT calculations demonstrating that hydrogenation of HCP regions leads to the most stable structures at low hydrogen coverage, whereas a mixed occupancy of both HCP and FCC regions becomes increasingly favorable at higher coverage. ARPES measurements were employed to investigate the change in electronic structure upon hydrogenation. It was found that a band gap can be opened in the graphene with a size controlled by the hydrogenation temperature. The formation of highly periodic hydrogen functionalization patterns on graphene revealed by STM in this study results in negligible relative broadening of the $\pi$ band, as observed by ARPES. The scattering rate of charge carriers in such modified graphene is therefore barely influenced by the presence of the well-ordered hydrogen clusters. These findings are of interest for employing graphene in electronic devices, where a tunable band gap along with preservation of the extraordinary electronic properties of graphene is required.

\section{METHODS}

$\operatorname{Ir}(111)$ was cleaned in UHV $\left(p<10^{-9} \mathrm{mbar}\right)$ by repeated cycles of $\mathrm{Ne}$ (STM) or Ar (XPS) sputtering at RT, followed by an anneal to 1470 $\mathrm{K}$. A monolayer of graphene was prepared by first forming graphene islands by $\mathrm{TPG}^{25}$ used for seeding followed by CVD to increase the coverage to a full monolayer. In more detail, an ethylene saturated $\operatorname{Ir}(111)$ surface was flashed to $1470 \mathrm{~K}$ (TPG), while CVD was done at $1270 \mathrm{~K}$ in $3 \times 10^{-6} \mathrm{mbar}$ of ethylene for $1 \mathrm{~min}$. This yields a full monolayer of high-quality graphene as seen in figure S1, where a large scale STM image is shown. The inset shows an atomically resolved image of the graphene structure. The large-scale modulation is a moire structure arising from the lattice mismatch between iridium and graphene. A submonolayer coverage of approximately 50\% (as seen in Figure 3B) was grown by three repetitive cycles of TPG.

Atomic hydrogen was deposited on the surface using a $2000 \mathrm{~K}$ hot hydrogen beam produced using a $\mathrm{HABS}^{37}$ atomic hydrogen source. Depositions were performed at a hydrogen background pressure of 3 $\times 10^{-7} \mathrm{mbar}$ for $3 \mathrm{~min}$ for the STM measurements. For the XPS measurements, the deposition time was $10 \mathrm{~min}$.

STM was performed at RT under UHV conditions on a commercial Createc STM. The atomic hydrogen depositions in this setup were performed at temperatures based on a type $\mathrm{K}$ thermocouple reading. The absolute temperature of these readings is subject to an uncertainty of approximately $\pm 15 \mathrm{~K}$, however, the relative temperature increments for the different depositions are accurate down to $1 \mathrm{~K}$.

XPS measurements were performed at beamline $\mathrm{I} 311^{38}$ at the MAX II laboratory in Lund. All C 1s spectra were acquired with a photon energy of $390 \mathrm{eV}$ in normal emission. Binding energies are calibrated to the Fermi level. The $\mathrm{C}$ 1s acquired before and after dosing hydrogen atoms at different temperatures were fitted with four Doniach-Š unjić components $\left(C_{a}, C_{b}, C_{c}\right.$ and $\left.C_{d}\right)$ convoluted with Gaussians. The Lorentzian full width at half-maximum was fixed to $0.16 \mathrm{eV}$ in our curve fitting procedure in agreement with previous studies, ${ }^{39}$ while the Gaussian full width at half-maximum (GFWHM) was allowed to change. To be consistent with previous curve fitting, ${ }^{27}$ the core level shifts of $\mathrm{C}_{\mathrm{a}}, \mathrm{C}_{\mathrm{b}}$, and $\mathrm{C}_{\mathrm{d}}$ components with respect to $\mathrm{C}_{\mathrm{c}}$ components were fixed to $+0.81 \mathrm{eV},+0.44 \mathrm{eV}$, and $-0.28 \mathrm{eV}$, respectively. To account for doping of the graphene film, the binding energy position the $\mathrm{C}_{\mathrm{c}}$ component was allowed to change. Data shown in Figure 4 of the main article are fitted by all-at-once fitting with one common GFWHM for the broad $\mathrm{C}_{\mathrm{a}}, \mathrm{C}_{\mathrm{b}}$, and $\mathrm{C}_{\mathrm{d}}$ components to reduce the parameter space. Our curve fitting reveals that the best fit is obtained with a GFWHM of $0.71 \mathrm{eV}$ for these components.

ARPES measurements were performed on the SGM3 end-station at the synchrotron radiation facility ASTRID2 in Aarhus. ${ }^{28}$ Graphene on $\operatorname{Ir}(111)$ was grown in a dedicated chamber using the standard procedures described above and then transferred in air to the endstation. Once in vacuum, the sample was annealed at $1000 \mathrm{~K}$ and then hydrogenated as described above. The ARPES data were acquired at $T_{\mathrm{s}}$ $=80 \mathrm{~K}$ using a photon energy of $47 \mathrm{eV}$ with an energy and angular resolution better than $20 \mathrm{meV}$ and $0.2^{\circ}$, respectively. In situ STM measurements using an Aarhus STM were performed to check the consistency of the preparation.

Band gap determination: Energy distribution curve (EDC) analysis has been performed to determine the top of the valence band using a Lorentzian function to fit each EDC. In the case of clean graphene, the Dirac point has been extrapolated from a linear fit of the ARPES accessible $\pi$ band.

$\pi$ Band broadening: Momentum distribution curve (MDC) analysis has been performed in order to determine the line width of the $\pi$ band identified as the full width at half-maximum obtained from a Lorentzian fit. The MDCs are taken at a binding energy $400 \mathrm{meV}$ below the extrapolated Dirac point. This binding energy has been chosen in order to avoid areas of the spectra where characteristic broadening due to phonons (at $200 \mathrm{meV}$ ) and minigaps (at $700 \mathrm{meV}$ ) occurs.

DFT was employed to atomically model the hydrogen atom adsorption onto graphene over the iridium surface. The real-space, grid-based projector augmented wave method GPAW was used ${ }^{40,41}$ in conjunction with the atomic simulation environment. ${ }^{42}$ See Supporting Information for additional computational information. 


\section{ASSOCIATED CONTENT}

\section{S Supporting Information}

The Supporting Information is available free of charge on the ACS Publications website at DOI: 10.1021/acsnano.6b04671.

Figures S1: computational methods; S2, STM images of clean graphene on $\operatorname{Ir}(111)$ after growth; S3, structures used for band dispersion calculations (PDF)

\section{AUTHOR INFORMATION}

\section{Corresponding Author}

*E-mail: liv@phys.au.dk.

\section{ORCID ${ }^{\circ}$}

Andrew Martin Cassidy: 0000-0001-8352-8721

Jan Knudsen: 0000-0002-8280-7638

Liv Hornekaer: 0000-0003-0828-3642

\section{Notes}

The authors declare no competing financial interest.

\section{ACKNOWLEDGMENTS}

We acknowledge Rocco Martinazzo for useful discussions and advise. The MAX IV Laboratory personnel are acknowledged for support during measurements. The Danish Council for Independent Research (grant no. 0602-02566B and grant no. 0602-02265B), Innovation Fund Denmark (NIAGRA, DAGATE), The European Research Council (CoG GRANN), The Swedish Research Council (project grant 2012-3850) are acknowledged for funding.

\section{REFERENCES}

(1) Schwierz, F. Graphene Transistors. Nat. Nanotechnol. 2010, 5, 487-496.

(2) Castro Neto, A. H.; Guinea, F.; Peres, N. M. R.; Novoselov, K. S.; Geim, A. K. The Electronic Properties of Graphene. Rev. Mod. Phys. 2009, 81, 109-162.

(3) Bolotin, K. I.; Sikes, K. J.; Jiang, Z.; Klima, M.; Fudenberg, G.; Hone, J.; Kim, P.; Stormer, H. L. Ultrahigh Electron Mobility in Suspended Graphene. Solid State Commun. 2008, 146, 351-355.

(4) Chen, Y. C.; de Oteyza, D. G.; Pedramrazi, Z.; Chen, C.; Fischer, F. R.; Crommie, M. F. Tuning the Band Gap of Graphene Nanoribbons Synthesized from Molecular Precursors. ACS Nano 2013, 7, 6123-6128.

(5) Ruffieux, P.; Cai, J. M.; Plumb, N. C.; Patthey, L.; Prezzi, D.; Ferretti, A.; Molinari, E.; Feng, X. L.; Mullen, K.; Pignedoli, C. A.; Fasel, R. Electronic Structure of Atomically Precise Graphene Nanoribbons. ACS Nano 2012, 6, 6930-6935.

(6) Zou, K.; Zhang, F.; Clapp, C.; MacDonald, A. H.; Zhu, J. Transport Studies of Dual-Gated ABC and ABA Trilayer Graphene: Band Gap Opening and Band Structure Tuning in Very Large Perpendicular Electric Fields. Nano Lett. 2013, 13, 369-373.

(7) Mak, K. F.; Lui, C. H.; Shan, J.; Heinz, T. F. Observation of an Electric-Field-Induced Band Gap in Bilayer Graphene by Infrared Spectroscopy. Phys. Rev. Lett. 2009, 102, 256405.

(8) Zhang, Y. B.; Tang, T. T.; Girit, C.; Hao, Z.; Martin, M. C.; Zettl, A.; Crommie, M. F.; Shen, Y. R.; Wang, F. Direct Observation of a Widely Tunable Bandgap in Bilayer Graphene. Nature 2009, 459, 820-823.

(9) Lu, Y. H.; Chen, W.; Feng, Y. P.; He, P. M. Tuning the Electronic Structure of Graphene by an Organic Molecule. J. Phys. Chem. B 2009, $113,2-5$.

(10) Yavari, F.; Kritzinger, C.; Gaire, C.; Song, L.; Gullapalli, H.; Borca-Tasciuc, T.; Ajayan, P. M.; Koratkar, N. Tunable Bandgap in Graphene by the Controlled Adsorption of Water Molecules. Small 2010, 6, 2535-2538.
(11) Plummer, J. D. Silicon VLSI Technology: Fundamentals, Practice, and Modeling; Pearson Education: India, 2009.

(12) Georgakilas, V.; Otyepka, M.; Bourlinos, A. B.; Chandra, V.; Kim, N.; Kemp, K. C.; Hobza, P.; Zboril, R.; Kim, K. S. Functionalization of Graphene: Covalent and Non-Covalent Approaches, Derivatives and Applications. Chem. Rev. 2012, 112, 61566214.

(13) Balog, R.; Jorgensen, B.; Nilsson, L.; Andersen, M.; Rienks, E.; Bianchi, M.; Fanetti, M.; Laegsgaard, E.; Baraldi, A.; Lizzit, S.; Sljivancanin, Z.; Besenbacher, F.; Hammer, B.; Pedersen, T. G.; Hofmann, P.; Hornekaer, L. Bandgap Opening in Graphene Induced by Patterned Hydrogen Adsorption. Nat. Mater. 2010, 9, 315-319.

(14) Haberer, D.; Vyalikh, D. V.; Taioli, S.; Dora, B.; Farjam, M.; Fink, J.; Marchenko, D.; Pichler, T.; Ziegler, K.; Simonucci, S.; Dresselhaus, M. S.; Knupfer, M.; Buchner, B.; Gruneis, A. Tunable Band Gap in Hydrogenated Quasi-Free-Standing Graphene. Nano Lett. 2010, 10, 3360-3366.

(15) Ulstrup, S.; Nilsson, L.; Miwa, J. A.; Balog, R.; Bianchi, M.; Hornekaer, L.; Hofmann, P. Electronic Structure of Graphene on a Reconstructed $\mathrm{Pt}(100)$ Surface: Hydrogen Adsorption, Doping, and Band Gaps. Phys. Rev. B: Condens. Matter Mater. Phys. 2013, 88, 125425.

(16) Kyhl, L.; Balog, R.; Angot, T.; Hornekær, L.; Bisson, R. Hydrogenated Graphene on $\operatorname{Ir}(111)$ : A High-Resolution Electron Energy Loss Spectroscopy Study of the Vibrational Spectrum. Phys. Rev. B: Condens. Matter Mater. Phys. 2016, 93, 115403.

(17) Garcia-Lastra, J. M. Strong Dependence of Band-Gap Opening at the Dirac Point of Graphene upon Hydrogen Adsorption Periodicity. Phys. Rev. B: Condens. Matter Mater. Phys. 2010, 82, 235418.

(18) Gao, X.; Wei, Z.; Meunier, V.; Sun, Y.; Zhang, S. B. Opening a Large Band Gap for Graphene by Covalent Addition. Chem. Phys. Lett. 2013, 555, 1-6.

(19) Kharche, N.; Nayak, S. K. Quasiparticle Band Gap Engineering of Graphene and Graphone on Hexagonal Boron Nitride Substrate. Nano Lett. 2011, 11, 5274-5278.

(20) Tang, S. B.; Yu, J. P.; Liu, L. X. Tunable Doping and Band Gap of Graphene on Functionalized Hexagonal Boron Nitride with Hydrogen and Fluorine. Phys. Chem. Chem. Phys. 2013, 15, 50675077.

(21) Elias, D. C.; Nair, R. R.; Mohiuddin, T. M. G.; Morozov, S. V.; Blake, P.; Halsall, M. P.; Ferrari, A. C.; Boukhvalov, D. W.; Katsnelson, M. I.; Geim, A. K.; Novoselov, K. S. Control of Graphene's Properties by Reversible Hydrogenation: Evidence for Graphane. Science 2009, $323,610-613$.

(22) Lebegue, S.; Klintenberg, M.; Eriksson, O.; Katsnelson, M. I. Accurate Electronic Band Gap of Pure and Functionalized Graphane from GW Calculations. Phys. Rev. B: Condens. Matter Mater. Phys. 2009, 79, 245117.

(23) Schulte, K.; Vinogradov, N. A.; Ng, M. L.; Martensson, N.; Preobrajenski, A. B. Bandgap Formation in Graphene on $\operatorname{Ir}(111)$ through Oxidation. Appl. Surf. Sci. 2013, 267, 74-76.

(24) Petersen, R.; Pedersen, T. G.; Jauho, A. P. Clar Sextet Analysis of Triangular, Rectangular, and Honeycomb Graphene Antidot Lattices. ACS Nano 2011, 5, 523-529.

(25) Coraux, J.; N’Diaye, A. T.; Engler, M.; Busse, C.; Wall, D.; Buckanie, N.; Heringdorf, F.; van Gastel, R.; Poelsema, B.; Michely, T. Growth of Graphene on $\operatorname{Ir}(111)$. New J. Phys. 2009, 11, 22.

(26) N'Diaye, A. T.; Coraux, J.; Plasa, T. N.; Busse, C.; Michely, T. Structure of Epitaxial Graphene on $\operatorname{Ir}(111)$. New J. Phys. 2008, 10, 043033 .

(27) Balog, R.; Andersen, M.; Jorgensen, B.; Sljivancanin, Z.; Hammer, B.; Baraldi, A.; Larciprete, R.; Hofmann, P.; Hornekaer, L.; Lizzit, S. Controlling Hydrogenation of Graphene on $\operatorname{Ir}(111)$. ACS Nano 2013, 7, 3823-3832.

(28) Hoffmann, S. V.; Sondergaard, C.; Schultz, C.; Li, Z.; Hofmann, P. An Undulator-Based Spherical Grating Monochromator Beamline for Angle-Resolved Photoemission Spectroscopy. Nucl. Instrum. Methods Phys. Res., Sect. A 2004, 523, 441-453. 
(29) Bostwick, A.; Ohta, T.; Seyller, T.; Horn, K.; Rotenberg, E. Quasiparticle Dynamics in Graphene. Nat. Phys. 2007, 3, 36-40.

(30) Pletikosic, I.; Kralj, M.; Pervan, P.; Brako, R.; Coraux, J.; N'Diaye, A. T.; Busse, C.; Michely, T. Dirac Cones and Minigaps for Graphene on $\operatorname{Ir}(111)$. Phys. Rev. Lett. 2009, 102, 056808.

(31) N’Diaye, A. T.; Gerber, T.; Busse, C.; Myslivecek, J.; Coraux, J.; Michely, T. A Versatile Fabrication Method for Cluster Superlattices. New J. Phys. 2009, 11, 103045.

(32) Pedersen, T. G.; Flindt, C.; Pedersen, J.; Mortensen, N. A.; Jauho, A. P.; Pedersen, K. Graphene Antidot Lattices: Designed Defects and Spin Qubits. Phys. Rev. Lett. 2008, 100, 189905.

(33) Grassi, R.; Low, T.; Lundstrom, M. Scaling of the Energy Gap in Pattern-Hydrogenated Graphene. Nano Lett. 2011, 11, 4574-4578.

(34) Hornekaer, L.; Sljivancanin, Z.; Xu, W.; Otero, R.; Rauls, E.; Stensgaard, I.; Laegsgaard, E.; Hammer, B.; Besenbacher, F. Metastable Structures and Recombination Pathways for Atomic Hydrogen on the Graphite (0001) Surface. Phys. Rev. Lett. 2006, 96, 156104.

(35) N’Diaye, A. T.; Bleikamp, S.; Feibelman, P. J.; Michely, T. TwoDimensional Ir Cluster Lattice on a Graphene Moire on $\operatorname{Ir}(111)$. Phys. Rev. Lett. 2006, 97, 215501.

(36) Zhou, S. Y.; Gweon, G. H.; Fedorov, A. V.; First, P. N.; De Heer, W. A.; Lee, D. H.; Guinea, F.; Castro Neto, A. H.; Lanzara, A. Substrate-induced bandgap opening in epitaxial graphene. Nat. Mater. 2007, 6, 916-916.

(37) Tschersich, K. G.; Fleischhauer, J. P.; Schuler, H. Design and Characterization of a Thermal Hydrogen Atom Source. J. Appl. Phys. 2008, 104, 034908.

(38) Nyholm, R.; Andersen, J. N.; Johansson, U.; Jensen, B. N.; Lindau, I. Beamline I311 at MAX-LAB: a VUV/Soft X-ray Undulator Beamline for High Resolution Electron Spectroscopy. Nucl. Instrum. Methods Phys. Res., Sect. A 2001, 467, 520-524.

(39) Granas, E.; Knudsen, J.; Schroder, U. A.; Gerber, T.; Busse, C.; Arman, M. A.; Schulte, K.; Andersen, J. N.; Michely, T. Oxygen Intercalation under Graphene on $\operatorname{Ir}(111)$ : Energetics, Kinetics, and the Role of Graphene Edges. ACS Nano 2012, 6, 9951-9963.

(40) Mortensen, J. J.; Hansen, L. B.; Jacobsen, K. W. Real-Space Grid Implementation of the Projector Augmented Wave Method. Phys. Rev. B: Condens. Matter Mater. Phys. 2005, 71, 035109.

(41) Enkovaara, J.; Rostgaard, C.; Mortensen, J. J.; Chen, J.; Dulak, M.; Ferrighi, L.; Gavnholt, J.; Glinsvad, C.; Haikola, V.; Hansen, H. A.; Kristoffersen, H. H.; Kuisma, M.; Larsen, A. H.; Lehtovaara, L.; Ljungberg, M.; Lopez-Acevedo, O.; Moses, P. G.; Ojanen, J.; Olsen, T.; Petzold, V.; et al. Electronic Structure Calculations with GPAW: a Real-Space Implementation of the Projector Augmented-Wave Method. J. Phys.: Condens. Matter 2010, 22, 253202.

(42) Bahn, S. R; Jacobsen, K. W. An Object-Oriented Scripting Interface to a Legacy Electronic Structure Code. Comput. Sci. Eng. 2002, 4, 56-66. 